\title{
On-axis non-linear effects with programmable Dammann lenses under femtosecond illumination
}

\author{
Jorge Pérez Vizcaíno, ${ }^{1, *}$ Omel Mendoza-Yero, ${ }^{1}$ Rocío Borrego-Varillas, ${ }^{1,2}$ Gladys Mínguez-Vega, ${ }^{1}$ \\ Javier R. Vázquez de Aldana, ${ }^{2}$ and Jesús Láncis ${ }^{1}$ \\ ${ }^{1}$ Instituto de Nuevas Tecnologías de la Imagen (INIT), Universidad Jaume I, Castellón E 12080, Spain \\ ${ }^{2}$ Departamento de Física Aplicada, Universidad de Salamanca, Salamanca, E-37008, Spain \\ ${ }^{*}$ Corresponding author: jvizcain@uji.es
}

Received February 8, 2013; revised April 10, 2013; accepted April 12, 2013; posted April 12, 2013 (Doc. ID 185116); published May 8, 2013

\begin{abstract}
We demonstrate the utilization of Dammann lenses codified onto a spatial light modulator (SLM) for triggering nonlinear effects. With continuous wave illumination Dammann lenses are binary phase optical elements that generate a set of equal intensity foci. We theoretically calculate the influence of ultrashort pulse illumination on the uniformity of the generated pattern, which is affected by chromatic aberration for pulses with temporal widths lower than $100 \mathrm{fs}$. The simulations also indicate that acceptable uniformity can be achieved for pulses of several fs by shortening the distance among foci which can be easily modified with the SLM. Multifocal second-harmonic generation (SHG) and on-axis multiple filamentation are produced and actively controlled in $\beta-\mathrm{BaB}_{2} \mathrm{O}_{4}(\mathrm{BBO})$ and fused silica samples, respectively, with an amplified Ti: Sapphire femtosecond laser of $30 \mathrm{fs}$ pulse duration. Experimental results are in very good agreement with theoretical calculations. (c) 2013 Optical Society of America OCIS codes: (050.1940) Diffraction; (320.6629) Supercontinuum generation; (320.7110) Ultrafast nonlinear optics. http://dx.doi.org/10.1364/OL.38.001621
\end{abstract}

The study of the non-linear processes [1] that take place during the interaction of light with matter has benefited from the high peak powers achieved with amplified femtosecond (fs) lasers [2]. This opens a huge gate toward the development of many applications in the nonlinear optics field. For instance, second-order parametric processes [3] are efficiently used to tune the central wavelength through SHG or sum/difference-frequency generation, expanding the accessible wavelength range of standard Ti:sapphire laser systems. Third-order processes are also very often exploited to increase the spectral width of fs pulses through optical-Kerr effect (self-phase modulation), or in combination with strongfield ionization and higher order processes (supercontinuum generation [4] and filamentation [5]). The increase of the spectral width by means of the abovementioned nonlinear effects allows subsequent compression of the pulse to a much shorter temporal duration [6].

Most of these processes require the focusing of the laser beam [7] just to reach the high intensities needed to trigger the desired effect. However, under certain circumstances, the spatiotemporal properties of the pulse focusing itself can be exploited to gain control over the nonlinear mechanisms taking place. In this sense, diffractive lenses have been recently employed for SHG [8] or in super continuum generation [9]: the strong chromatic aberration induced by diffraction changes the spectral properties of the pulse.

On the other hand, Dammann diffraction gratings are binary phase distributions of alternate $0, \pi$ zones for well-defined transient points [10,11]. For continuous wave $(\mathrm{CW})$ illumination, these gratings generate diffraction patterns characterized by a number $N$ of diffracted orders with the same peak intensity. This concept can be easily extended to lenses. The phase of a converging refractive lens can be represented by $\pi r^{2} / \lambda f$, where $r$ denotes the radial coordinate, $f$ the focal length, and $\lambda$ the wavelength of light. The lens function $\exp \left(i \pi r^{2} / \lambda f\right)$ can be binarized by applying the Dammann grating formalism to the squared radial coordinate $r^{2}$ [12]. The resultant binary lens function will create a series of $N$ focal planes having focal lengths of $f / n$ with equal peak intensities, where $n=(\ldots,-3,-1,1,3, \ldots)$ for an even number of orders, and $n=(\ldots,-2,-1,0,1,2, \ldots)$ for an odd number of orders [13]. Although Dammann lenses encoded into a spatial light modulator (SLM) can be a powerful tool for dynamic control and generation of nonlinear effects, they have not been explored yet in this context.

In this letter, we first summarize the characteristics of the on-axis diffraction pattern for Dammann lenses encoded into an SLM illuminated by ultrashort laser sources. Second, two results from experiments are shown to prove its potential to trigger nonlinear effects. Specifically, we demonstrate the application of Dammann lenses to SHG and filamentation in transparent dielectrics.

In Fig. 1 a schematic diagram of the optical setup is shown. For the experiment we use a Ti:Sapphire fs laser that emits linearly polarized pulses of about $30 \mathrm{fs}$ at intensity FWHM, centered at $\lambda_{0}=800 \mathrm{~nm}$, with $50 \mathrm{~nm}$ of spectral bandwidth and a repetition rate of $1 \mathrm{kHz}$. Dammann lenses are encoded onto a reflective liquid

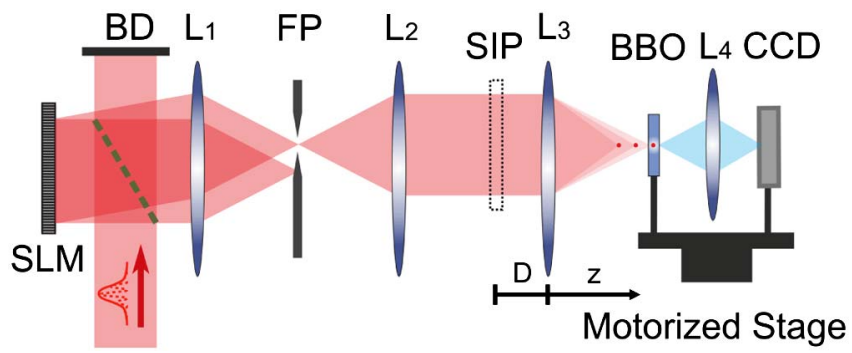

Fig. 1. Experimental setup for the analysis of nonlinear effects caused by multiple on-axis foci generated with Dammann lenses encoded into an SLM. The input beam is split in two rays by a beam splitter (green dotted line) to work with the SLM at normal incidence. A beam dumper (BD) is used to block the unused part of the beam. 
crystal on silicon (LCoS) SLM (Holoeye Pluto). The SLM has $1920 \times 1080$ pixels with $8 \mu \mathrm{m}$ of pixel pitch. The reflected beam from the SLM passes through a $1 \times$ telescope composed of a pair of lenses $\mathrm{L}_{1}$ and $\mathrm{L}_{2}$ of focal lengths $f_{1}=f_{2}=100 \mathrm{~mm}$. The telescope makes an image of the SLM plane after the lens $\mathrm{L}_{2}$. To avoid the zero diffraction order of the SLM, we overlap a linear phase of period $0.3 \mathrm{~mm}$ to the Dammann lens, and use in the experiment its first diffraction order.

In addition, to ensure an equal spacing among all Dammann foci, a third lens $\mathrm{L}_{3}$ is placed at the focal length distance $f_{3}=50 \mathrm{~mm}$ from the SLM image plane (SIP). This configuration allows us to obtain a number $N$ of equally spaced focused spots with the same peak irradiance. The focal planes $\left(z=f_{n}\right.$ from $\left.\mathrm{L}_{3}\right)$ of the foci can be determined by the expression

$$
\frac{1}{f_{n}}=\frac{1}{F}-\frac{n}{n D-f}
$$

From Eq. (1), it follows that there are three parameters to control the output of our optical system: the number of foci (related to the parameter $n$ ), the focal length of Dammann lenses, and the focal length of the refractive lens $L_{3}$. The axial width of the foci can be determined by solving the Huygens-Fresnel diffraction equation in the ABCD matrix formalism. To do that, we calculate the irradiance distribution along the propagation axis $z$ when no phase is encoded into the SLM (it can be regarded as a mirror), and thus a collimated laser beam reaches the refractive lens $\mathrm{L}_{3}$, i.e., when $n=0$. In this condition, the normalized on-axis irradiance can be determined by the expression

$$
I(z)=\frac{\sin ^{2}\left(\frac{A \pi a^{2}}{\lambda z}\right)}{A^{2}},
$$

where $A=1-z / F$ and a is the radius of the encoded Dammann lens. At this point we analyze the influence of broadband light spectra on the irradiance given by Dammann lenses under ultrashort pulsed illumination. In the bottom part of Fig. $\underline{2}$ the Fresnel diffraction integral is used to simulate the irradiance $I(z)$. Here, the onaxis irradiance obtained with a Dammann lens of 5 equal foci separated by $2.05 \mathrm{~mm}$ is shown. As it can be seen in the top part of Fig. 2, the simulation was carried out for (a)
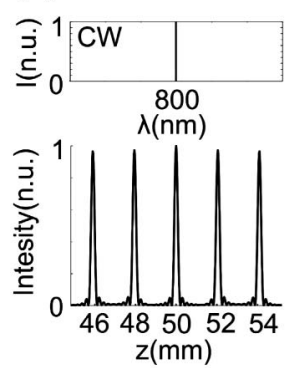

(b)

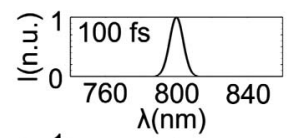

(c)
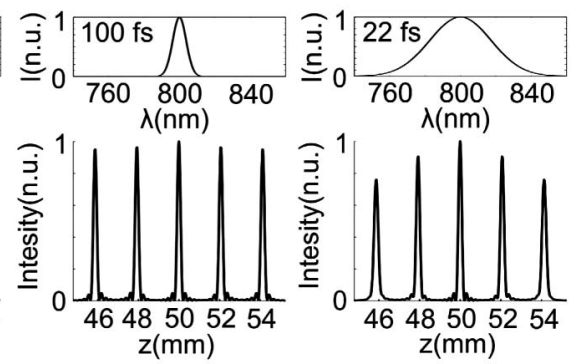

Fig. 2. Simulated irradiance patterns obtained with a Dammann lens, which is illuminated with (a) monochromatic beam, (b) 100 fs laser pulse, and (c) 22 fs laser pulse. two different Fourier transform limited Gaussian laser pulses, and an ideal CW source.

The irradiance uniformity in the case of the CW source is approximately $10^{-5}$ [11]. Small discrepancies in the height of the peaks are caused mainly due to deviations of the $0-\pi$ transition locations from their ideal values when encoding Dammann lenses into an SLM $[14,15]$. For an ultrashort laser pulse of 100 fs falls of $0.3 \%$ and $1.5 \%$ (for $n= \pm 1$ and $n= \pm 2$ orders, respectively) are registered. The equivalent values for 22 fs pulses were $6 \%$ and $20 \%$. In accordance to our simulations, for laser pulses close to or greater than $100 \mathrm{fs}$, chromatic aberrations do not influence excessively the uniformity of Damman irradiance patterns. In contrast, for shorter pulses (below a few tens of fs), the effects of chromatic aberrations cannot be neglected e.g., see Fig. 2(c). In addition, we found out that the longer the separation between foci, the greater the reduction of the irradiance uniformity due to chromatic aberrations. Therefore, chromatic aberrations will increase the spatial width and decrease the peak irradiance of the foci, reducing the uniformity of the generated irradiance patterns. The efficiency of this experiment is mainly affected by the SLM device efficiency and the inherent Dammann lens efficiency. Taking all into account the estimated efficiency of the system is of about $40 \%$.

In order to show the potential of Dammann lenses to manage non-linear effects, we developed a couple of experiments. First, we explored SHG originated by a programmable set of on-axis Dammann foci. To do that, a type-I $\beta-\mathrm{BaB}_{2} \mathrm{O}_{4}(\mathrm{BBO})$ crystal $\left(\theta=29.2^{\circ}, 20 \mu \mathrm{m}\right.$ thickness) was displaced along the different foci generated with Dammann lenses in the focal region of $\mathrm{L}_{3}$. With the help of the lens $\mathrm{L}_{4}$, magnified images of each focus were formed onto a charge-coupled device (CCD) camera. To avoid transmission of infrared light a suitable filter was placed in front of the CCD. The scanning device (made up of BBO crystal, lens $\mathrm{L}_{4}$, and CCD) was mounted on a motorized stage, which allows for computer controlled movements via Matlab code. The thickness of the non-linear crystal ensures enough spectral acceptance for the SHG of the fundamental pulses, which energy was carefully set to avoid the damage of the crystal during the scan.

In Fig. 3, experimental results are shown. Furthermore, images of the transversal focal spots, the spectrum

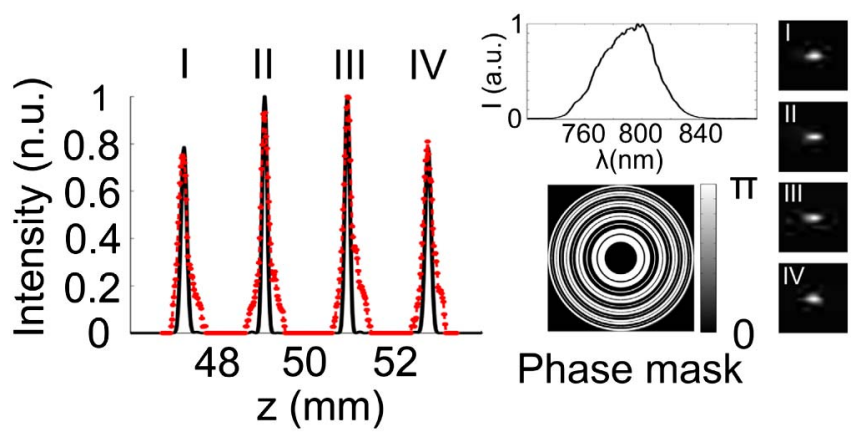

Fig. 3. On-axis multifocal SHG with Dammann lenses: experiment (red dotted line) and theory (black line). Dammann phase mask, pulse spectrum, and images of the foci from a lateral side of the non-linear crystal are shown as insets. 
of our laser, and the encoded Dammann phase mask are provided as insets. This Dammann phase mask generates a set of $\mathrm{N}=4$ foci separated by $1.92 \mathrm{~mm}$. The motor step was set to $20 \mu \mathrm{m}$, whereas the focal length of $\mathrm{L}_{3}$ was $\mathrm{F}=\mathrm{D}=50 \mathrm{~mm}$. The second harmonic irradiance recorded with the CCD is plotted as a function of the distance $z$ from the lens $\mathrm{L}_{3}$, and represented by a red dotted line. Within the paraxial Fresnel approximation, the experimental results are compared to numerical simulations (black line) of the squared normalized irradiance $I^{2}(z)$ calculated for the measured spectrum of our laser. From a visual inspection of Fig. $\underline{3}$ it is apparent that experimental results are in good agreement with the simulations. We believe that almost inappreciable differences in the locations of the peak irradiances are mainly due to possible deviations of the pulsed laser beam (toward convergence behavior) from its collimated state before the $\mathrm{L}_{3}$ lens. In addition, the increase in the axial width of experimental data with respect to simulations is mainly attributed to the discrepancy of our beam from the plane wave illumination considered in simulations. Misalignment can also influence the broadening of the foci slightly.

In the second experiment, we take advantage of programmable Dammann lenses to produce multiple spatially tuned filaments in a fused silica sample. The motorized stage was removed and a fused silica sample $(20 \mathrm{~mm} \times 10 \mathrm{~mm} \times 5 \mathrm{~mm})$ with all faces polished was placed in the focal region of $\mathrm{L}_{3}$ lens $(\mathrm{F}=\mathrm{D}=50 \mathrm{~mm})$. Then, the pulse energy was progressively increased from 0 to the minimum value at which filaments were observed in the sample at the various foci of the lens. The plasma emission of the filaments was registered in a CCD from one of the sides of the sample. The number of foci, as well as the distance between them, was controlled to produce several filaments with different Dammann phase masks. The corresponding images are shown in Fig. 4. Moreover, the yellow lines show the on-axis irradiance profiles along the whole extension of the filaments. In particular, Fig. 4(a) and 4(b) correspond to four filaments with separations of 2.1 and $0.4 \mathrm{~mm}$, respectively. In Fig. 4(c) three filaments separated by $2.14 \mathrm{~mm}$ are generated, whereas in Fig. 4(d) the number of filaments is increased to six with a distance among them of $0.85 \mathrm{~mm}$. As it might be expected both the shape and intensity of the filaments change with their distance from the air-glass interface due to the spherical aberration induced by light refraction $[16,17]$.

We have shown that Dammann lenses can be used as a powerful tool to manipulate "at will" certain non-linear effects with intense fs pulses. Hence, several applications might be expected e.g., for performing in-depth parallel processing of transparent dielectrics $[\underline{18}, \underline{19}]$. In this paper we have investigated on-axis Dammann lenses, but our formalism can be easily extended to other cases (e.g., bidimensional Dammann structures).
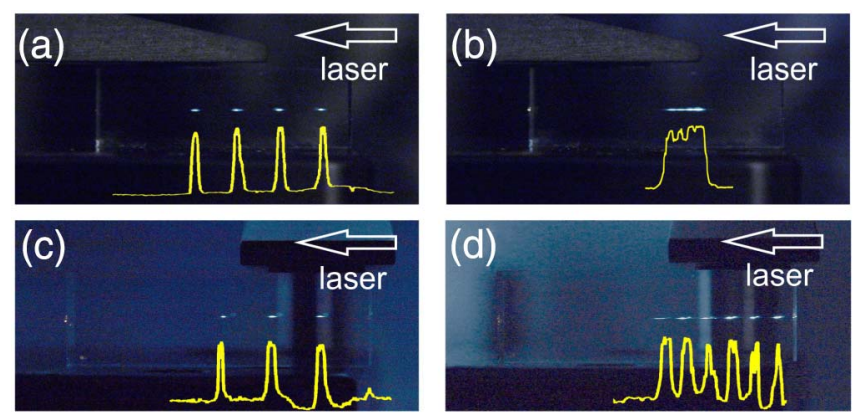

Fig. 4. Examples of multifilament generation in fused silica glass. The number of filaments and distances between them are changed by encoding different Dammann lenses.

This research was funded by the Spanish Ministerio de Ciencia e Innovación through Consolider Programme (SAUUL CSD2007-00013) and FIS2010-15746. Also, financial support from the Generalitat Valenciana through project PROMETEO/2012/021 and Pla Propi de Investigació de la Universitat Jaume I through project P11B201026 is acknowledged. Authors are also very grateful to the SCIC of the Universitat Jaume I for the use of the femtosecond laser.

\section{References}

1. R. W. Boyd, Nonlinear Optics (Academic, 2008).

2. D. Strickland and G. Mourou, Opt. Commun. 56, 219 (1985).

3. P. Franken, A. Hill, C. Peters, and G. Weinreich, Phys. Rev. Lett. 7, 118 (1961).

4. R. Alfano, The Supercontinuum Laser Source (Springer, 2006).

5. A. Couairon and A. Mysyrowicz, Phys. Rep. 441, 47 (2007).

6. M. Nisoli, S. De Silvestri, and O. Svelto, Appl. Phys. Lett. 68, 2793 (1996).

7. G. Boyd and D. Kleinman, J. Appl. Phys. 39, 3597 (1968).

8. G. Mínguez-Vega, C. Romero, O. Mendoza-Yero, J. R. Vázquez de Aldana, R. Borrego-Varillas, C. Méndez, P. Andrés, J. Lancis, and L. Roso, Opt. Lett. 35, 3694 (2010).

9. C. Romero, R. Borrego-Varillas, A. Camino, G. MinguezVega, O. Mendoza-Yero, J. Hernandez-Toro, and J. R. Vázquez de Aldana, Opt. Express 19, 4977 (2011).

10. H. Dammann and E. Klotz, Opt. Acta 24, 505 (1977).

11. C. Zhou and L. Liu, Appl. Opt. 34, 5961 (1995).

12. I. Moreno, J. A. Davis, D. M. Cottrell, N. Zhang, and X.-C. Yuan, Opt. Lett. 35, 1536 (2010).

13. J. A. Davis, I. Moreno, J. L. Martínez, T. J. Hernández, and D. M. Cottrell, Appl. Opt. 50, 3653 (2011).

14. J. Yu, C. Zhou, W. Jia, W. Cao, S. Wang, J. Ma, and H. Cao, Appl. Opt. 51, 1619 (2012).

15. D. C. O’Shea, Appl. Opt. 34, 6533 (1995).

16. N. Huot, R. Stoian, A. Mermillod-Blondin, C. Mauclair, and E. Audouard, Opt. Express 15, 12395 (2007).

17. Q. Sun, H. Jiang, Y. Liu, Y. Zhou, H. Yang, and Q. Gong, J. Opt. A 7, 655 (2005).

18. R. Gattass and E. Mazur, Nat. Photonics 2, 219 (2008).

19. C. Mauclair, A. Mermillond-Blondin, S. Landon, N. Huot, A. Rosenfeld, I. V. Hertel, E. Audouard, I. Myiamoto, and R. Stoian, Opt. Lett. 36, 325 (2011). 